\title{
Evaluation challenges in large-scale document summarization
}

\section{Dragomir R. Radev}

U. of Michigan

radev@umich.edu

Wai Lam

Chinese U. of Hong Kong

wlam@se.cuhk.edu.hk

\author{
Simone Teufel \\ U. of Cambridge \\ simone.teufel@cl.cam.ac.uk \\ John Blitzer \\ U. of Pennsylvania \\ blitzer@ seas.upenn.edu
}

\author{
Danyu Liu \\ U. of Alabama \\ liudy@cis.uab.edu
}

\author{
Horacio Saggion \\ U. of Sheffield \\ h.saggion@dcs.shef.ac.uk
}

\author{
Arda Çelebi \\ ardax@isi.edu
}

\begin{abstract}
We present a large-scale meta evaluation of eight evaluation measures for both single-document and multi-document summarizers. To this end we built a corpus consisting of (a) 100 Million automatic summaries using six summarizers and baselines at ten summary lengths in both English and Chinese, (b) more than 10,000 manual abstracts and extracts, and (c) 200 Million automatic document and summary retrievals using 20 queries. We present both qualitative and quantitative results showing the strengths and drawbacks of all evaluation methods and how they rank the different summarizers.
\end{abstract}

\section{Introduction}

Automatic document summarization is a field that has seen increasing attention from the NLP community in recent years. In part, this is because summarization incorporates many important aspects of both natural language understanding and natural language generation. In part it is because effective automatic summarization would be useful in a variety of areas. Unfortunately, evaluating automatic summarization in a standard and inexpensive way is a difficult task (Mani et al., 2001). Traditional large-scale evaluations are either too simplistic (using measures like precision, recall, and percent agreement which (1) don't take chance agreement into account and (2) don't account for the fact that human judges don't agree which sentences should be in a summary) or too expensive (an approach using manual judgements can scale up to a few hundred summaries but not to tens or hundreds of thousands).

In this paper, we present a comparison of six summarizers as well as a meta-evaluation including eight measures: Precision/Recall, Percent Agreement, Kappa, Relative Utility, Relevance Correlation, and three types of Content-Based measures (cosine, longest common subsequence, and word overlap). We found that while all measures tend to rank summarizers in different orders, measures like Kappa, Relative Utility, Relevance Correlation and Content-Based each offer significant advantages over the more simplistic methods.

\section{Data, Annotation, and Experimental Design}

We performed our experiments on the Hong Kong News corpus provided by the Hong Kong SAR of the People's Republic of China (LDC catalog number LDC2000T46). It contains 18,146 pairs of parallel documents in English and Chinese. The texts are not typical news articles. The Hong Kong Newspaper mainly publishes announcements of the local administration and descriptions of municipal events, such as an anniversary of the fire department, or seasonal festivals. We tokenized the corpus to identify headlines and sentence boundaries. For the English text, we used a lemmatizer for nouns and verbs. We also segmented the Chinese documents using the tool provided at http://www.mandarintools.com.

Several steps of the meta evaluation that we performed involved human annotator support. First, we 


\begin{tabular}{|l|l|}
\hline Cluster 2 & Meetings with foreign leaders \\
Cluster 46 & Improving Employment Opportunities \\
Cluster 54 & Illegal immigrants \\
Cluster 60 & Customs staff doing good job. \\
Cluster 61 & Permits for charitable fund raising \\
Cluster 62 & Y2K readiness \\
Cluster 112 & Autumn and sports carnivals \\
Cluster 125 & Narcotics Rehabilitation \\
Cluster 199 & Intellectual Property Rights \\
Cluster 241 & Fire safety, building management concerns \\
Cluster 323 & Battle against disc piracy \\
Cluster 398 & Flu results in Health Controls \\
Cluster 447 & Housing (Amendment) Bill Brings Assorted Improvements \\
Cluster 551 & Natural disaster victims aided \\
Cluster 827 & Health education for youngsters \\
Cluster 885 & Customs combats contraband/dutiable cigarette operations \\
Cluster 883 & Public health concerns cause food-business closings \\
Cluster 1014 & Traffic Safety Enforcement \\
Cluster 1018 & Flower shows \\
Cluster 1197 & Museums: exhibits/hours \\
\hline
\end{tabular}

Figure 1: Twenty queries created by the LDC for this experiment.

asked LDC to build a set of queries (Figure 1). Each of these queries produced a cluster of relevant documents. Twenty of these clusters were used in the experiments in this paper.

Additionally, we needed manual summaries or extracts for reference. The LDC annotators produced summaries for each document in all clusters. In order to produce human extracts, our judges also labeled sentences with "relevance judgements", which indicate the relevance of sentence to the topic of the document. The relevance judgements for sentences range from 0 (irrelevant) to 10 (essential). As in (Radev et al., 2000), in order to create an extract of a certain length, we simply extract the top scoring sentences that add up to that length.

For each target summary length, we produce an extract using a summarizer or baseline. Then we compare the output of the summarizer or baseline with the extract produced from the human relevance judgements. Both the summarizers and the evaluation measures are described in greater detail in the next two sections.

\subsection{Summarizers and baselines}

This section briefly describes the summarizers we used in the evaluation. All summarizers take as input a target length (n\%) and a document (or cluster) split into sentences. Their output is an $n \%$ extract of the document (or cluster).

- MEAD (Radev et al., 2000): MEAD is a centroid-based extractive summarizer that scores sentences based on sentence-level and inter-sentence features which indicate the quality of the sentence as a summary sentence. It then chooses the top-ranked sentences for inclusion in the output summary. MEAD runs on both English documents and on BIG5-encoded Chinese. We tested the summarizer in both languages.

- WEBS (Websumm (Mani and Bloedorn, 2000)): can be used to produce generic and query-based summaries. Websumm uses a graph-connectivity model and operates under the assumption that nodes which are connected to many other nodes are likely to carry salient information.

- SUMM (Summarist (Hovy and Lin, 1999)): an extractive summarizer based on topic signatures.

- ALGN (alignment-based): We ran a sentence alignment algorithm (Gale and Church, 1993) for each pair of English and Chinese stories. We used it to automatically generate Chinese "manual" extracts from the English manual extracts we received from LDC.

- LEAD (lead-based): n\% sentences are chosen from the beginning of the text.

- RAND (random): $\mathrm{n} \%$ sentences are chosen at random.

The six summarizers were run at ten different target lengths to produce more than 100 million summaries (Figure 2). For the purpose of this paper, we only focus on a small portion of the possible experiments that our corpus can facilitate.

\section{Summary Evaluation Techniques}

We used three general types of evaluation measures: co-selection, content-based similarity, and relevance correlation. Co-selection measures include precision and recall of co-selected sentences, relative utility (Radev et al., 2000), and Kappa (Siegel and Castellan, 1988; Carletta, 1996). Co-selection methods have some restrictions: they only work for extractive summarizers. Two manual summaries of the same input do not in general share many identical sentences. We address this weakness of co-selection 


\begin{tabular}{|c|c|c|c|c|c|c|c|c|c|c|c|c|}
\hline & \multicolumn{11}{|c|}{$\begin{array}{l}\text { Lengths } \\
\end{array}$} & \multirow[t]{2}{*}{$\# \mathrm{dj}$} \\
\hline & $05 \mathrm{~W}$ & $05 \mathrm{~S}$ & $10 \mathrm{~W}$ & $10 \mathrm{~S}$ & $20 \mathrm{~W}$ & $20 \mathrm{~S}$ & $30 \mathrm{~W}$ & $30 \mathrm{~S}$ & $40 \mathrm{~W}$ & $40 \mathrm{~S}$ & FD & \\
\hline E-FD & - & - & - & - & - & - & - & - & - & - & $\mathrm{x}$ & 40 \\
\hline E-LD & $\mathrm{X}$ & $\mathrm{X}$ & $\mathrm{X}$ & $\mathrm{X}$ & $\mathrm{x}$ & $\mathrm{x}$ & $\mathrm{X}$ & $\mathrm{X}$ & $\mathrm{X}$ & $\mathrm{X}$ & - & 440 \\
\hline E-RA & $\mathrm{X}$ & $\mathrm{X}$ & $\mathrm{X}$ & $\mathrm{X}$ & $\mathrm{x}$ & $\mathrm{x}$ & $\mathrm{X}$ & $\mathrm{X}$ & $\mathrm{X}$ & $\mathrm{X}$ & - & 440 \\
\hline E-MO & $\mathrm{x}$ & $\mathrm{x}$ & $\mathrm{X}$ & $\mathrm{x}$ & $\mathrm{x}$ & $\mathrm{x}$ & $\mathrm{X}$ & $\mathrm{x}$ & $\mathrm{X}$ & $\mathrm{x}$ & - & 540 \\
\hline E-M2 & - & - & - & - & - & $\mathrm{X}$ & - & - & - & - & - & 20 \\
\hline E-M3 & - & - & - & - & - & $\mathrm{X}$ & - & - & - & - & - & 8 \\
\hline E-S2 & - & - & - & - & - & $\mathrm{X}$ & - & - & - & - & - & 8 \\
\hline E-WS & - & $\mathrm{X}$ & - & $\mathrm{X}$ & $\mathrm{x}$ & $\mathrm{x}$ & - & $\mathrm{X}$ & - & $\mathrm{X}$ & - & 160 \\
\hline E-WQ & - & - & - & - & - & $\mathrm{X}$ & - & - & - & - & - & 10 \\
\hline E-LC & - & - & - & - & - & - & $\mathrm{x}$ & - & - & - & - & 40 \\
\hline E-CY & - & $\mathrm{X}$ & - & $\mathrm{X}$ & - & $\mathrm{x}$ & - & $\mathrm{X}$ & - & $\mathrm{X}$ & - & 120 \\
\hline E-AL & $\mathrm{X}$ & $\mathrm{X}$ & $\mathrm{X}$ & $\mathrm{X}$ & $\mathrm{X}$ & $\mathrm{X}$ & $\mathrm{X}$ & $\mathrm{X}$ & $\mathrm{X}$ & $\mathrm{X}$ & - & 200 \\
\hline E-AR & $\mathrm{X}$ & $\mathrm{X}$ & $\mathrm{X}$ & $\mathrm{X}$ & $\mathrm{X}$ & $\mathrm{X}$ & $\mathrm{X}$ & $\mathrm{X}$ & $\mathrm{X}$ & $\mathrm{X}$ & - & 200 \\
\hline E-AM & $\mathrm{X}$ & $\mathrm{X}$ & $\mathrm{X}$ & $\mathrm{X}$ & $\mathrm{X}$ & $\mathrm{X}$ & $\mathrm{X}$ & $\mathrm{X}$ & $\mathrm{X}$ & $\mathrm{X}$ & - & 200 \\
\hline C-FD & - & - & - & - & - & - & - & - & - & - & $\mathrm{x}$ & 40 \\
\hline C-LD & $\mathrm{X}$ & $\mathrm{X}$ & $\mathrm{X}$ & $\mathrm{X}$ & $\mathrm{x}$ & $\mathrm{x}$ & $\mathrm{X}$ & $\mathrm{X}$ & $\mathrm{X}$ & $\mathrm{X}$ & - & 240 \\
\hline C-RA & $\mathrm{X}$ & $\mathrm{X}$ & $\mathrm{X}$ & X & $\mathrm{x}$ & $\mathrm{x}$ & $\mathrm{X}$ & $\mathrm{X}$ & $\mathrm{X}$ & $\mathrm{X}$ & - & 240 \\
\hline C-MO & $\mathrm{X}$ & $\mathrm{x}$ & $\mathrm{X}$ & $\mathrm{x}$ & $\mathrm{x}$ & $\mathrm{x}$ & $\mathrm{X}$ & $\mathrm{x}$ & $\mathrm{X}$ & $\mathrm{x}$ & - & 320 \\
\hline C-M2 & - & - & - & - & - & $\mathrm{X}$ & - & - & - & - & - & 20 \\
\hline $\mathrm{C}-\mathrm{CY}$ & - & $\mathrm{X}$ & - & $\mathrm{X}$ & - & $\mathrm{x}$ & - & $\mathrm{X}$ & - & $\mathrm{X}$ & - & 120 \\
\hline C-AL & $\mathrm{X}$ & $\mathrm{X}$ & $\mathrm{X}$ & $\mathrm{X}$ & $\mathrm{X}$ & $\mathrm{X}$ & $\mathrm{X}$ & $\mathrm{X}$ & $\mathrm{X}$ & $\mathrm{X}$ & - & 180 \\
\hline C-AR & $\mathrm{X}$ & $\mathrm{X}$ & $\mathrm{X}$ & $\mathrm{X}$ & $\mathrm{X}$ & $\mathrm{X}$ & $\mathrm{X}$ & $\mathrm{X}$ & $\mathrm{X}$ & $\mathrm{X}$ & - & 200 \\
\hline C-AM & - & $\mathrm{X}$ & $\mathrm{X}$ & $\mathrm{X}$ & $\mathrm{X}$ & $\mathrm{X}$ & $\mathrm{X}$ & $\mathrm{X}$ & & $\mathrm{X}$ & - & 120 \\
\hline X-FD & - & - & - & - & - & - & - & - & - & - & $\mathrm{x}$ & 40 \\
\hline X-LD & $\mathrm{X}$ & $\mathrm{X}$ & $\mathrm{X}$ & $\mathrm{X}$ & $x$ & $x$ & $\mathrm{X}$ & $\mathrm{X}$ & $\mathrm{X}$ & $\mathrm{X}$ & - & 240 \\
\hline X-RA & $\mathrm{X}$ & $\mathrm{X}$ & $\mathrm{X}$ & $\mathrm{X}$ & $\mathrm{x}$ & $\mathrm{x}$ & $\mathrm{X}$ & $\mathrm{X}$ & $\mathrm{X}$ & $\mathrm{X}$ & - & 240 \\
\hline X-MO & $\mathrm{X}$ & $\mathrm{x}$ & $\mathrm{X}$ & $\mathrm{x}$ & $\mathrm{x}$ & $\mathrm{x}$ & $\mathrm{X}$ & $\mathrm{x}$ & $\mathrm{X}$ & $\mathrm{x}$ & - & 320 \\
\hline X-M2 & - & - & - & - & - & $\mathrm{X}$ & - & - & - & - & - & 20 \\
\hline $\mathrm{X}-\mathrm{CY}$ & - & $\mathrm{X}$ & - & $\mathrm{X}$ & - & $\mathrm{x}$ & - & $\mathrm{X}$ & - & $\mathrm{X}$ & - & 120 \\
\hline X-AL & $\mathrm{X}$ & $\mathrm{X}$ & $\mathrm{X}$ & $\mathrm{X}$ & $\mathrm{X}$ & $\mathrm{X}$ & $\mathrm{X}$ & $\mathrm{X}$ & $\mathrm{X}$ & $\mathrm{X}$ & - & 140 \\
\hline X-AR & $\mathrm{X}$ & $\mathrm{X}$ & $\mathrm{X}$ & $\mathrm{X}$ & $\mathrm{X}$ & $\mathrm{X}$ & $\mathrm{X}$ & $\mathrm{X}$ & $\mathrm{X}$ & $\mathrm{X}$ & - & 160 \\
\hline X-AM & - & $\mathrm{X}$ & $\mathrm{X}$ & $\mathrm{X}$ & $\mathrm{X}$ & $\mathrm{X}$ & $\mathrm{X}$ & $\mathrm{X}$ & - & $\mathrm{X}$ & - & 120 \\
\hline
\end{tabular}

Figure 2: All runs performed ( $\mathrm{X}=20$ clusters, $x=10$ clusters $)$. Language: $\mathrm{E}=$ English, $\mathrm{C}=\mathrm{Chinese}$, $\mathrm{X}=$ cross-lingual; Summarizer: $\mathrm{LD}=\mathrm{LEAD}, \mathrm{RA}=\mathrm{RAND}, \mathrm{WS}=\mathrm{WEBS}, \mathrm{WQ}=\mathrm{WEBS}$-query based, etc.; $\mathrm{S}=$ sentence-based, $\mathrm{W}$ = word-based; \#dj = number of "docjudges" (ranked lists of documents and summaries). Target lengths above $50 \%$ are not shown in this table for lack of space. Each run is available using two different retrieval schemes. We report results using the cross-lingual retrievals in a separate paper.

measures with several content-based similarity measures. The similarity measures we use are word overlap, longest common subsequence, and cosine.

One advantage of similarity measures is that they can compare manual and automatic extracts with manual abstracts. To our knowledge, no systematic experiments about agreement on the task of summary writing have been performed before. We use similarity measures to measure interjudge agreement among three judges per topic. We also apply the measures between human extracts and summaries, which answers the question if human extracts are more similar to automatic extracts or to human summaries.

The third group of evaluation measures includes relevance correlation. It shows the relative performance of a summary: how much the performance of document retrieval decreases when indexing summaries rather than full texts.

Task-based evaluations (e.g., SUMMAC (Mani et al., 2001), DUC (Harman and Marcu, 2001), or (Tombros et al., 1998) measure human performance using the summaries for a certain task (after the summaries are created). Although they can be a very effective way of measuring summary quality, task-based evaluations are prohibitively expensive at large scales. In this project, we didn't perform any task-based evaluations as they would not be appropriate at the scale of millions of summaries.

\subsection{Evaluation by sentence co-selection}

For each document and target length we produce three extracts from the three different judges, which we label throughout as $J 1, J 2$, and $J 3$.

We used the rates $5 \%, 10 \%, 20 \%, 30 \%, 40 \%$ for most experiments. For some experiments, we also consider summaries of $50 \%, 60 \%, 70 \%, 80 \%$ and $90 \%$ of the original length of the documents. Figure 3 shows some abbreviations for co-selection that we will use throughout this section.

\subsubsection{Precision and Recall}

Precision and recall are defined as:

$$
P_{J_{2}}\left(J_{1}\right)=\frac{A}{A+C}, R_{J_{2}}\left(J_{1}\right)=\frac{A}{A+B}
$$




\begin{tabular}{|c|c|c|c|c|}
\hline & \multicolumn{2}{|c|}{$J_{2}$} & \\
\hline & & $\begin{array}{l}\text { Sentence in } \\
\text { Extract }\end{array}$ & $\begin{array}{l}\text { Sentence not } \\
\text { in Extract }\end{array}$ & \\
\hline \multirow{3}{*}{$J_{1}$} & $\begin{array}{l}\text { Sentence in } \\
\text { Extract }\end{array}$ & $A$ & $B$ & $A+B$ \\
\hline & $\begin{array}{l}\text { Sentence not } \\
\text { in Extract }\end{array}$ & C & $D$ & $C+D$ \\
\hline & & $A+C$ & $B+D$ & $\begin{array}{l}N=A+ \\
B+C+D\end{array}$ \\
\hline
\end{tabular}

Figure 3: Contingency table comparing sentences extracted by the system and the judges.

In our case, each set of documents which is compared has the same number of sentences and also the same number of sentences are extracted; thus $P=R$.

The average precision $P_{\text {avg }}(S Y S T E M)$ and recall $R_{\text {avg }}(S Y S T E M)$ are calculated by summing over individual judges and normalizing. The average interjudge precision and recall is computed by averaging over all judge pairs.

However, precision and recall do not take chance agreement into account. The amount of agreement one would expect two judges to reach by chance depends on the number and relative proportions of the categories used by the coders. The next section on Kappa shows that chance agreement is very high in extractive summarization.

\subsubsection{Kappa}

Kappa (Siegel and Castellan, 1988) is an evaluation measure which is increasingly used in NLP annotation work (Krippendorff, 1980; Carletta, 1996). Kappa has the following advantages over P and R:

- It factors out random agreement. Random agreement is defined as the level of agreement which would be reached by random annotation using the same distribution of categories as the real annotators.

- It allows for comparisons between arbitrary numbers of annotators and items.

- It treats less frequent categories as more important (in our case: selected sentences), similarly to precision and recall but it also considers (with a smaller weight) more frequent categories as well.

The Kappa coefficient controls agreement $P(A)$ by taking into account agreement by chance $P(E)$ :

$$
K=\frac{P(A)-P(E)}{1-P(E)}
$$

No matter how many items or annotators, or how the categories are distributed, $K=0$ when there is no agreement other than what would be expected by chance, and $K=1$ when agreement is perfect. If two annotators agree less than expected by chance, Kappa can also be negative.

We report Kappa between three annotators in the case of human agreement, and between three humans and a system (i.e. four judges) in the next section.

\subsubsection{Relative Utility}

Relative Utility (RU) (Radev et al., 2000) is tested on a large corpus for the first time in this project. RU takes into account chance agreement as a lower bound and interjudge agreement as an upper bound of performance. RU allows judges and summarizers to pick different sentences with similar content in their summaries without penalizing them for doing so. Each judge is asked to indicate the importance of each sentence in a cluster on a scale from 0 to 10. Judges also specify which sentences subsume or paraphrase each other. In relative utility, the score of an automatic summary increases with the importance of the sentences that it includes but goes down with the inclusion of redundant sentences.

\subsection{Content-based Similarity measures}

Content-based similarity measures compute the similarity between two summaries at a more finegrained level than just sentences. For each automatic extract $S$ and similarity measure $M$ we compute the following number:

$$
\operatorname{sim}(M, S,\{J 1, J 2, J 3\})=\frac{M(S, J 1)+M(S, J 2)+M(S, J 3)}{3}
$$

We used several content-based similarity measures that take into account different properties of the text:

Cosine similarity is computed using the following formula (Salton, 1988):

$$
\cos (X, Y)=\frac{\sum x_{i} * y_{i}}{\sqrt{\sum\left(x_{i}\right)^{2}} * \sqrt{\sum\left(y_{i}\right)^{2}}}
$$


where $X$ and $Y$ are text representations based on the vector space model.

Longest Common Subsequence is computed as follows:

$$
\operatorname{lcs}(X, Y)=(\text { length }(X)+\text { length }(Y)-d(X, Y)) / 2
$$

where $X$ and $Y$ are representations based on sequences and where $l c s(X, Y)$ is the length of the longest common subsequence between $X$ and $Y$, length $(X)$ is the length of the string $X$, and $d(X, Y)$ is the minimum number of deletion and insertions needed to transform $X$ into $Y$ (Crochemore and Rytter, 1994).

\subsection{Relevance Correlation}

Relevance correlation ( $\mathrm{RC}$ ) is a new measure for assessing the relative decrease in retrieval performance when indexing summaries instead of full documents. The idea behind it is similar to (Sparck-Jones and Sakai, 2001). In that experiment, Sparck-Jones and Sakai determine that short summaries are good substitutes for full documents at the high precision end. With RC we attempt to rank all documents given a query.

Suppose that given a query $Q$ and a corpus of documents $D_{i}$, a search engine ranks all documents in $D_{i}$ according to their relevance to the query $Q$. If instead of the corpus $D_{i}$, the respective summaries of all documents are substituted for the full documents and the resulting corpus of summaries $S_{i}$ is ranked by the same retrieval engine for relevance to the query, a different ranking will be obtained. If the summaries are good surrogates for the full documents, then it can be expected that rankings will be similar.

There exist several methods for measuring the similarity of rankings. One such method is Kendall's tau and another is Spearman's rank correlation. Both methods are quite appropriate for the task that we want to perform; however, since search engines produce relevance scores in addition to rankings, we can use a stronger similarity test, linear correlation between retrieval scores. When two identical rankings are compared, their correlation is 1 . Two completely independent rankings result in a score of 0 while two rankings that are reverse versions of one another have a score of -1 . Although rank correlation seems to be another valid measure, given the large number of irrelevant documents per query resulting in a large number of tied ranks, we opted for linear correlation. Interestingly enough, linear correlation and rank correlation agreed with each other.

Relevance correlation $r$ is defined as the linear correlation of the relevance scores ( $x$ and $y$ ) assigned by two different IR algorithms on the same set of documents or by the same IR algorithm on different data sets:

$$
r=\frac{\sum_{i}\left(x_{i}-\bar{x}\right)\left(y_{i}-\bar{y}\right)}{\sqrt{\sum_{i}\left(x_{i}-\bar{x}\right)^{2}} \sqrt{\sum_{i}\left(y_{i}-\bar{y}\right)^{2}}}
$$

Here $\bar{x}$ and $\bar{y}$ are the means of the relevance scores for the document sequence.

We preprocess the documents and use Smart to index and retrieve them. After the retrieval process, each summary is associated with a score indicating the relevance of the summary to the query. The relevance score is actually calculated as the inner product of the summary vector and the query vector. Based on the relevance score, we can produce a full ranking of all the summaries in the corpus.

In contrast to (Brandow et al., 1995) who run 12 Boolean queries on a corpus of 21,000 documents and compare three types of documents (full documents, lead extracts, and ANES extracts), we measure retrieval performance under more than 300 conditions (by language, summary length, retrieval policy for 8 summarizers or baselines).

\section{Results}

This section reports results for the summarizers and baselines described above. We relied directly on the relevance judgements to create "manual extracts" to use as gold standards for evaluating the English systems. To evaluate Chinese, we made use of a table of automatically produced alignments. While the accuracy of the alignments is quite high, we have not thoroughly measured the errors produced when mapping target English summaries into Chinese. This will be done in future work.

\subsection{Co-selection results}

Co-selection agreement (Section 3.1) is reported in Figures 4, and 5). The tables assume human performance is the upper bound, the next rows compare the different summarizers. 
Figure 4 shows results for precision and recall. We observe the effect of a dependence of the numerical results on the length of the summary, which is a well-known fact from information retrieval evaluations.

Websumm has an advantage over MEAD for longer summaries but not for $20 \%$ or less. Lead summaries perform better than all the automatic summarizers, and better than the human judges. This result usually occurs when the judges choose different, but early sentences. Human judgements overtake the lead baseline for summaries of length $50 \%$ or more.

\begin{tabular}{|c|c|c|c|c|c|}
\hline & $\mathbf{5 \%}$ & $\mathbf{1 0 \%}$ & $\mathbf{2 0 \%}$ & $\mathbf{3 0 \%}$ & $\mathbf{4 0 \%}$ \\
\hline Humans & .187 & .246 & .379 & .467 & .579 \\
\hline MEAD & .160 & .231 & .351 & .420 & .519 \\
\hline WEBS & .310 & .305 & .358 & .439 & .543 \\
\hline LEAD & .354 & .387 & .447 & .483 & .583 \\
\hline RAND & .094 & .113 & .224 & .357 & .432 \\
\hline
\end{tabular}

Figure 4: Results in precision=recall (averaged over 20 clusters).

Figure 5 shows results using Kappa. Random agreement is 0 by definition between a random process and a non-random process.

While the results are overall rather low, the numbers still show the following trends:

- MEAD outperforms Websumm for all but the $5 \%$ target length.

- Lead summaries perform best below 20\%, whereas human agreement is higher after that.

- There is a rather large difference between the two summarizers and the humans (except for the 5\% case for Websumm). This numerical difference is relatively higher than for any other co-selection measure treated here.

- Random is overall the worst performer.

- Agreement improves with summary length.

Figures 6 and 7 summarize the results obtained through Relative Utility. As the figures indicate, random performance is quite high although all nonrandom methods outperform it significantly. Further, and in contrast with other co-selection evaluation criteria, in both the single- and multi-document

\begin{tabular}{|l|c|c|c|c|c|}
\hline & $\mathbf{5 \%}$ & $\mathbf{1 0 \%}$ & $\mathbf{2 0 \%}$ & $\mathbf{3 0 \%}$ & $\mathbf{4 0 \%}$ \\
\hline Humans & .127 & .157 & .194 & .225 & .274 \\
\hline MEAD & .109 & .136 & .168 & .192 & .230 \\
\hline WEBS & .138 & .128 & .146 & .159 & .192 \\
\hline LEAD & .180 & .198 & .213 & .220 & .261 \\
\hline RAND & .064 & .081 & .097 & .116 & .137 \\
\hline
\end{tabular}

Figure 5: Results in kappa, averaged over 20 clusters.

case MEAD outperforms LEAD for shorter summaries (5-30\%). The lower bound (R) represents the average performance of all extracts at the given summary length while the upper bound $(\mathrm{J})$ is the interjudge agreement among the three judges.

\begin{tabular}{|c|c|c|c|c|c|}
\hline & $\mathbf{5 \%}$ & $\mathbf{1 0 \%}$ & $\mathbf{2 0 \%}$ & $\mathbf{3 0 \%}$ & $\mathbf{4 0 \%}$ \\
\hline R & 0.66 & 0.68 & 0.71 & 0.74 & 0.76 \\
\hline RAND & 0.67 & 0.67 & 0.71 & 0.75 & 0.77 \\
\hline WEBS & 0.72 & 0.73 & 0.76 & 0.79 & 0.82 \\
\hline LEAD & 0.72 & 0.73 & 0.77 & 0.80 & 0.83 \\
\hline MEAD & 0.78 & 0.79 & 0.79 & 0.81 & 0.83 \\
\hline J & 0.80 & 0.81 & 0.83 & 0.85 & 0.87 \\
\hline
\end{tabular}

Figure 6: RU per summarizer and summary length (Single-document).

\begin{tabular}{|c|c|c|c|c|c|}
\hline & $\mathbf{5 \%}$ & $\mathbf{1 0 \%}$ & $\mathbf{2 0 \%}$ & $\mathbf{3 0 \%}$ & $\mathbf{4 0 \%}$ \\
\hline R & 0.64 & 0.66 & 0.69 & 0.72 & 0.74 \\
\hline RAND & 0.63 & 0.65 & 0.71 & 0.72 & 0.74 \\
\hline LEAD & 0.71 & 0.71 & 0.76 & 0.79 & 0.82 \\
\hline MEAD & 0.73 & 0.75 & 0.78 & 0.79 & 0.81 \\
\hline J & 0.76 & 0.78 & 0.81 & 0.83 & 0.85 \\
\hline
\end{tabular}

Figure 7: RU per summarizer and summary length (Multi-document).

\subsection{Content-based results}

The results obtained for a subset of target lengths using content-based evaluation can be seen in Figures 8 and 9. In all our experiments with $t f * i d f$ weighted cosine, the lead-based summarizer obtained results close to the judges in most of the target lengths while MEAD is ranked in second position. In all our experiments using longest common subsequence, no system obtained better results in the majority of the cases.

\begin{tabular}{|l|c|c|c|c|}
\hline & $\mathbf{1 0 \%}$ & $\mathbf{2 0 \%}$ & $\mathbf{3 0 \%}$ & $\mathbf{4 0 \%}$ \\
\hline LEAD & 0.55 & 0.65 & 0.70 & 0.79 \\
\hline MEAD & 0.46 & 0.61 & 0.70 & 0.78 \\
\hline RAND & 0.31 & 0.47 & 0.60 & 0.69 \\
\hline WEBS & 0.52 & 0.60 & 0.68 & 0.77 \\
\hline
\end{tabular}

Figure 8: Cosine $(t f * i d f)$. Average over 10 clusters. 


\begin{tabular}{|l|c|c|c|c|}
\hline & $\mathbf{1 0 \%}$ & $\mathbf{2 0 \%}$ & $\mathbf{3 0 \%}$ & $\mathbf{4 0 \%}$ \\
\hline LEAD & 0.47 & 0.55 & 0.60 & 0.70 \\
\hline MEAD & 0.37 & 0.52 & 0.61 & 0.70 \\
\hline RAND & 0.25 & 0.38 & 0.50 & 0.58 \\
\hline WEBS & 0.39 & 0.45 & 0.53 & 0.64 \\
\hline
\end{tabular}

Figure 9: Longest Common Subsequence. Average over 10 clusters.

The numbers obtained in the evaluation of Chinese summaries for cosine and longest common subsequence can be seen in Figures 10 and 11. Both measures identify MEAD as the summarizer that produced results closer to the ideal summaries (these results also were observed across measures and text representations).

\begin{tabular}{|l|c|c|c|c|}
\hline & $\mathbf{1 0 \%}$ & $\mathbf{2 0 \%}$ & $\mathbf{3 0 \%}$ & $\mathbf{4 0 \%}$ \\
\hline SUMM & 0.44 & 0.65 & 0.71 & 0.78 \\
\hline LEAD & 0.54 & 0.63 & 0.68 & 0.77 \\
\hline MEAD & 0.49 & 0.65 & 0.74 & 0.82 \\
\hline RAND & 0.31 & 0.50 & 0.65 & 0.71 \\
\hline
\end{tabular}

Figure 10: Chinese Summaries. Cosine $(t f * i d f)$. Average over 10 clusters. Vector space of Words as Text Representation.

\begin{tabular}{|l|c|c|c|c|}
\hline & $\mathbf{1 0 \%}$ & $\mathbf{2 0 \%}$ & $\mathbf{3 0 \%}$ & $\mathbf{4 0 \%}$ \\
\hline SUMM & 0.32 & 0.53 & 0.57 & 0.65 \\
\hline LEAD & 0.42 & 0.49 & 0.54 & 0.64 \\
\hline MEAD & 0.35 & 0.50 & 0.60 & 0.70 \\
\hline RAND & 0.21 & 0.35 & 0.49 & 0.54 \\
\hline
\end{tabular}

Figure 11: Chinese Summaries. Longest Common Subsequence. Average over 10 clusters. Chinese Words as Text Representation.

We have based this evaluation on target summaries produced by LDC assessors, although other alternatives exist. Content-based similarity measures do not require the target summary to be a subset of sentences from the source document, thus, content evaluation based on similarity measures can be done using summaries published with the source documents which are in many cases available (Teufel and Moens, 1997; Saggion, 2000).

\subsection{Relevance Correlation results}

We present several results using Relevance Correlation. Figures 12 and 13 show how RC changes depending on the summarizer and the language used. $\mathrm{RC}$ is as high as 1.0 when full documents (FD) are compared to themselves. One can notice that even random extracts get a relatively high RC score. It is also worth observing that Chinese summaries score lower than their corresponding English summaries. Figure 14 shows the effects of summary length and summarizers on RC. As one might expect, longer summaries carry more of the content of the full document than shorter ones. At the same time, the relative performance of the different summarizers remains the same across compression rates.

\begin{tabular}{|l|l|l|l|l|l|l|}
\hline & C112 & C125 & C241 & C323 & C551 & AVG10 \\
\hline FD & 1.00 & 1.00 & 1.00 & 1.00 & 1.00 & 1.000 \\
MEAD & 0.91 & 0.92 & 0.93 & 0.92 & 0.90 & 0.903 \\
WEBS & 0.88 & 0.82 & 0.89 & 0.91 & 0.88 & 0.843 \\
LEAD & 0.80 & 0.80 & 0.84 & 0.85 & 0.81 & 0.802 \\
RAND & 0.80 & 0.78 & 0.87 & 0.85 & 0.79 & 0.800 \\
SUMM & 0.77 & 0.79 & 0.85 & 0.88 & 0.81 & 0.775 \\
\hline
\end{tabular}

Figure 12: RC per summarizer (English 20\%).

\begin{tabular}{|l|l|l|l|l|l|l|}
\hline & C112 & C125 & C241 & C323 & C551 & AVG10 \\
\hline FD & 1.00 & 1.00 & 1.00 & 1.00 & 1.00 & 1.000 \\
MEAD & 0.78 & 0.87 & 0.93 & 0.66 & 0.91 & 0.850 \\
SUMM & 0.76 & 0.75 & 0.85 & 0.84 & 0.75 & 0.755 \\
RAND & 0.71 & 0.75 & 0.85 & 0.60 & 0.74 & 0.744 \\
ALGN & 0.74 & 0.72 & 0.83 & 0.95 & 0.72 & 0.738 \\
LEAD & 0.72 & 0.71 & 0.83 & 0.58 & 0.75 & 0.733 \\
\hline
\end{tabular}

Figure 13: RC per summarizer (Chinese, 20\%).

\begin{tabular}{|l|l|l|l|l|l|}
\hline & $\mathbf{5 \%}$ & $\mathbf{1 0 \%}$ & $\mathbf{2 0 \%}$ & $\mathbf{3 0 \%}$ & $\mathbf{4 0 \%}$ \\
\hline FD & 1.000 & 1.000 & 1.000 & 1.000 & 1.000 \\
MEAD & 0.724 & 0.834 & 0.916 & 0.946 & 0.962 \\
WEBS & 0.730 & 0.804 & 0.876 & 0.912 & 0.936 \\
LEAD & 0.660 & 0.730 & 0.820 & 0.880 & 0.906 \\
SUMM & 0.622 & 0.710 & 0.820 & 0.848 & 0.862 \\
RAND & 0.554 & 0.708 & 0.818 & 0.884 & 0.922 \\
\hline
\end{tabular}

Figure 14: RC per summary length and summarizer.

\section{Conclusion}

This paper describes several contributions to text summarization:

First, we observed that different measures rank summaries differently, although most of them showed that "intelligent" summarizers outperform lead-based summaries which is encouraging given that previous results had cast doubt on the ability of summarizers to do better than simple baselines.

Second, we found that measures like Kappa, Relative Utility, Relevance Correlation and ContentBased, each offer significant advantages over more simplistic methods like Precision, Recall, and Percent Agreement with respect to scalability, applicability to multidocument summaries, and ability to include human and chance agreement. Figure 15 


\begin{tabular}{|c|c|c|c|c|c|}
\hline Property & Prec, recall & Kappa & Normalized RU & Word overlap, cosine, LCS & Relevance Correlation \\
\hline Intrinsic (I)/extrinsic (E) & I & I & I & I & $\mathrm{E}$ \\
\hline Agreement between human extracts & $\mathrm{X}$ & $\mathrm{X}$ & $\mathrm{X}$ & $\mathrm{X}$ & $\mathrm{X}$ \\
\hline Agreement human extracts and automatic extracts & $\mathrm{X}$ & $\mathrm{X}$ & $\mathrm{X}$ & $\mathrm{X}$ & $\mathrm{X}$ \\
\hline Agreement human abstracts and human extracts & & & & $\bar{X}$ & \\
\hline Non-binary decisions & & & $\mathrm{X}$ & $\mathrm{X}$ & \\
\hline Takes random agreement into account by design & & $\mathrm{X}$ & $\mathrm{X}$ & & \\
\hline Full documents vs. extracts & & & & $\mathrm{X}$ & $\mathrm{X}$ \\
\hline Systems with different sentence segmentation & & & & $\mathrm{X}$ & $\bar{X}$ \\
\hline Multidocument extracts & $\mathrm{X}$ & $\mathrm{X}$ & $\mathrm{X}$ & $\mathrm{X}$ & \\
\hline Full corpus coverage & & & & $\mathrm{X}$ & $\mathrm{X}$ \\
\hline
\end{tabular}

Figure 15: Properties of evaluation measures used in this project.

presents a short comparison of all these evaluation measures.

Third, we performed extensive experiments using a new evaluation measure, Relevance Correlation, which measures how well a summary can be used to replace a document for retrieval purposes.

Finally, we have packaged the code used for this project into a summarization evaluation toolkit and produced what we believe is the largest and most complete annotated corpus for further research in text summarization. The corpus and related software is slated for release by the LDC in mid 2003.

\section{References}

Ron Brandow, Karl Mitze, and Lisa F. Rau. 1995. Automatic Condensation of Electronic Publications by Sentence Selection. Information Processing and Management, 31(5):675-685.

Jean Carletta. 1996. Assessing Agreement on Classification Tasks: The Kappa Statistic. CL, 22(2):249-254.

Maxime Crochemore and Wojciech Rytter. 1994. Text Algorithms. Oxford University Press.

William A. Gale and Kenneth W. Church. 1993. A program for aligning sentences in bilingual corpora. Computational Linguistics, 19(1):75-102.

Donna Harman and Daniel Marcu, editors. 2001. Proceedings of the 1st Document Understanding Conference. New Orleans, LA, September.

Eduard Hovy and Chin Yew Lin. 1999. Automated Text Summarization in SUMMARIST. In Inderjeet Mani and Mark T. Maybury, editors, Advances in Automatic Text Summarization, pages 81-94. The MIT Press.

Klaus Krippendorff. 1980. Content Analysis: An Introduction to its Methodology. Sage Publications, Beverly Hills, CA.

Inderjeet Mani and Eric Bloedorn. 2000. Summarizing Similarities and Differences Among Related Documents. Information Retrieval, 1(1).
Inderjeet Mani, Thérèse Firmin, David House, Gary Klein, Beth Sundheim, and Lynette Hirschman. 2001. The TIPSTER SUMMAC Text Summarization Evaluation. In Natural Language Engineering.

Dragomir R. Radev, Hongyan Jing, and Malgorzata Budzikowska. 2000. Centroid-Based Summarization of Multiple Documents: Sentence Extraction, UtilityBased Evaluation, and User Studies. In Proceedings of the Workshop on Automatic Summarization at the 6th Applied Natural Language Processing Conference and the 1st Conference of the North American Chapter of the Association for Computational Linguistics, Seattle, WA, April.

Horacio Saggion. 2000. Génération automatique de résumés par analyse sélective. Ph.D. thesis, Département d'informatique et de recherche opérationnelle. Faculté des arts et des sciences. Université de Montréal, August.

Gerard Salton. 1988. Automatic Text Processing. Addison-Wesley Publishing Company.

Sidney Siegel and N. John Jr. Castellan. 1988. Nonparametric Statistics for the Behavioral Sciences. McGraw-Hill, Berkeley, CA, 2nd edition.

Karen Sparck-Jones and Tetsuya Sakai. 2001. Generic Summaries for Indexing in IR. In Proceedings of the 24th Annual International ACM SIGIR Conference on Research and Development in Information Retrieval, pages 190-198, New Orleans, LA, September.

Simone Teufel and Marc Moens. 1997. Sentence Extraction as a Classification Task. In Proceedings of the Workshop on Intelligent Scalable Text Summarization at the 35th Meeting of the Association for Computational Linguistics, and the 8th Conference of the European Chapter of the Assocation for Computational Linguistics, Madrid, Spain.

Anastasios Tombros, Mark Sanderson, and Phil Gray. 1998. Advantages of Query Biased Summaries in Information Retrieval. In Eduard Hovy and Dragomir R. Radev, editors, Proceedings of the AAAI Symposium on Intelligent Text Summarization, pages 34-43, Stanford, California, USA, March 23-25,. The AAAI Press. 